\title{
THEORETICAL ACCURACY PREDICTION AND VALIDATION OF LOW-END AND HIGH-END MOBILE MAPPING SYSTEM IN URBAN, RESIDENTIAL AND RURAL AREAS
}

\author{
L. Mattheuwsen*, M. Bassier, M. Vergauwen \\ Dept. of Civil Engineering, TC Construction - Geomatics \\ KU Leuven - Faculty of Engineering Technology \\ Ghent, Belgium \\ (lukas.mattheuwsen,maarten.bassier,maarten.vergauwen)@kuleuven.be
}

\section{Technical Commission II}

KEY WORDS: accuracy prediction, accuracy analysis, mobile mapping system, low-end, high-end

\begin{abstract}
:
Mobile mapping systems are increasingly being used for the acquisition of 3D information of the environment. Although these systems are very efficient in data capturing compared to more traditional methods, the high cost of high-end accurate mobile mapping systems is a major drawback. In contrast, the much cheaper low-end mobile mapping systems are more frequently used for less accurate projects where visualization is more important. In general, the achievable accuracy level is the driving factor that differentiates low-end from high-end systems. To determine this value, the sensor quality, calibration and GNSS reception quality should be reliably evaluated.

In this paper, we present a theoretical accuracy model of a mobile mapping system that takes into account variable GNSS accuracy. The predicted accuracy level of low-end and high-end mobile mapping systems is evaluated in a comprehensive accuracy analysis. The absolute accuracy of the system is determined in three datasets in which GNSS reception quality varies between optimal, good and poor. Additionally, the relative accuracy of both systems is checked by comparison of control distances. The presented approach allows for a more general and robust accuracy prediction of mobile mapping systems in different circumstances.
\end{abstract}

\section{INTRODUCTION}

In the last decade, Mobile Mapping Systems (MMS) have proven to be among the most efficient methods of capturing large scale georeferenced 3D data of large areas. Compared to other techniques such as Terrestrial Laser Scanning (TLS) or traditional surveying techniques, MMS are able to achieve similar results and accuracy levels in a much shorter timespan. Because of these advantages, MMS are deployed in numerous applications such as large scale mapping (Hwang et al., 2013), road asset management (Sairam et al., 2016), coastal monitoring (Bitenc et al., 2011) and damage assessment (Ajmar et al., 2013).

While the first MMS system was developed almost 3 decades ago, the general layout and operating principal has remained the same. A MMS is divided into two sub-systems, a positioning and a mapping system (Puente et al., 2013a). The positioning system uses a Global Navigation Satellite System (GNSS) for the absolute positioning of the MMS. Additionally, an Inertial Measuring Unit (IMU) and Distance Measurement Indicator (DMI) measure the rotation angles and the travelled distance respectively. By combining the data of th GNSS, IMU and DMI in a kalman filter, the SBET (Smoothed Best Estimated Trajectory) for the MMS is computed. The mapping system performs the acquisition of spatial data and typically consists of one or more lidar sensors and (omni-directional) cameras. The precise calibration of both sub-systems results in accurate georeferenced imagery and lidar data.

The applicability of a MMS predominantly depends on the required accuracy level. For mobile mapping systems, a common

\footnotetext{
*Corresponding author
}

distinction is made between "survey-grade" MMS and "mappinggrade" MMS (Kersting, Friess, 2016). The former uses highend sensors to achieve high accuracy levels for engineering or design projects. In contrast, the latter is equipped with a lowend IMU and lidar sensors for mapping or visualisation purposes. There is a significant price difference between both systems with high-end systems costing up to 5 or even 10 times more than low-end systems. It is therefore imperative to choose the proper system for an application.

The choice of MMS in an application is driven by both the absolute and relative accuracy level of the system which depend on the quality and calibration of the configured sensors. These accuracy levels can be predicted by a theoretical accuracy model using the general working principal of MMS and the parameters of the configured sensors such as done in (Schaer et al., 2007, Barber et al., 2008, Goel, Lohani, 2015, Craig et al., 2007). In order to validate the theoretical model, an accuracy study of the system is conducted in real-world circumstances such as performed in (Hofmann, Brenner, 2016, Schaer et al., 2007, Toschi et al., 2015, Puente et al., 2013b, Kaartinen et al., 2012, Hauser et al., 2016, Barber et al., 2008). Typically, these theoretical models use fixed general accuracies for the positioning sensors instead of modelling a variable accuracy for GNSS. Additionally, the validation is generally performed in areas that are considered to be good circumstances for GNSS. However, to completely validate a MMS, these studies should be performed in varying circumstances.

In our method, we present a more complete accuracy analysis of mobile mapping systems compared to other studies. The theoretical accuracy model includes a varying accuracy for the GNSS 


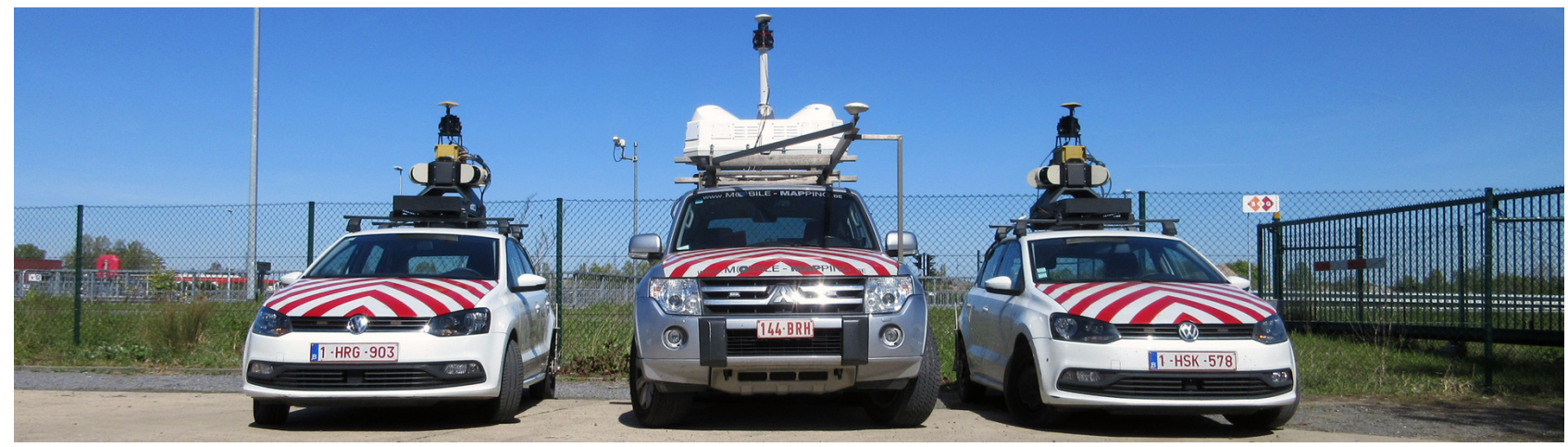

Figure 1. Example Mobile Mapping Systems: IP-S2 Compact system (left and right) and Lynx mobile mapper system (centre).

and is validated in three datasets with varying circumstances in terms of GNSS reception. The experiments are performed on both a "mapping-grade" low-end system and a "surveyinggrade" high-end system.

The remainder of this paper is organized as follows. Section 2 provides the related work on the accuracy prediction and analysis of MMS. Section 3 presents our methodology. In Section 4 the test datasets are discussed. In Section 5 the experimental results are presented. Finally, the conclusions are presented in Section 6.

\section{RELATED WORK}

As stated above, the decision to use a mobile mapping system for an application is mainly determined by the achievable accuracy. This accuracy depends on the accuracy of the two sub-systems of a MMS. On the one hand, the positioning system measures the absolute position of the mapping frame in a coordinate system and determines the external accuracy of the system. On the other hand, the mapping system captures the environment with lidar or image sensors and defines the internal or relative accuracy of the system. The combination of the two results in the absolute accuracy of a mobile mapping system and predicts the expected errors in the georeferenced data in a certain global or projected coordinate system. The influence of both accuracies is illustrated in Figure 2. Low positioning and high mapping accuracy results in accurate distances between buildings but coordinates being shifted in a certain direction. In contrast, with high positioning and low relative accuracy, the MMS is positioned correctly but the mapping measurements result in a noisy and inaccurate representation of the environment. Only with high positioning and high mapping accuracy, high absolute accuracy is achieved in order to correctly capture the environment.

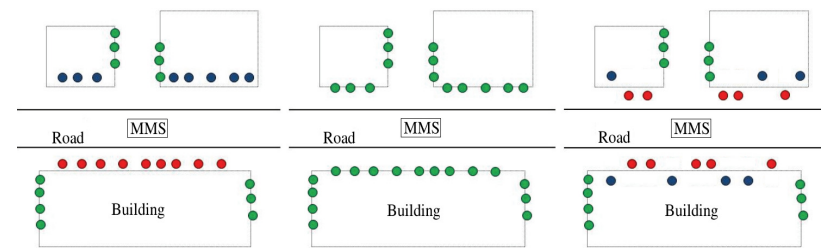

Figure 2. Illustration of the influence of positioning and mapping (relative) accuracy on the absolute accuracy of a MMS: low pos. + high map. accuracy (left), high pos. + high map. accuracy (centre) and high pos. + low map. accuracy (right).

The absolute and relative accuracy depend on the accuracy of positioning sensors, mapping sensors and the calibration between them. Optimizing the "accuracy to cost" ratio becomes important to achieve the required accuracy and at the same time reduce the total cost of the system. Knowing the theoretical achievable accuracy and influence of each sensor is essential before a purchasing decision. Using the general working principal of a mobile mapping system, the absolute and relative accuracy can be predicted based on the accuracy of the configured sensors. Research done in (Barber et al., 2008, Goel, Lohani, 2015) analyses the influence of the parameters on the absolute accuracy of a low-end and high-end mobile mapping system. It is concluded that for both low-end (Barber et al., 2008) and high-end systems (Goel, Lohani, 2015), the GNSS/IMU positioning accuracy is the driving factor of the absolute accuracy of a mobile mapping system. Therefore, in order to achieve the highest possible accuracy level, the main focus should be on a robust, reliable and accurate positioning system. In our method, both the absolute and relative accuracy of low-end and high-end systems are computed based on the sensor configuration of the system. Additionally, a variable parameter for GNSS reception quality is implemented in order to accurately predict the theoretical absolute accuracy of mobile mapping systems in more challenging circumstances.

In order to quantify the predicted accuracy of a mobile mapping system, accuracy studies are conducted in real-world circumstances. In (Barber et al., 2008), a MMS with low-end lidar sensors is analysed in terms of elevation and planimetric accuracy. The system is tested in a residential and industrial area where tall buildings and narrow streets are present. The comparison with GCP (Ground Control Points) collected with RTK GNSS resulted in a RMS error in elevation of $0.03 \mathrm{~m}$ as predicted with the theoretical model. In contrast, the planimetric accuracy was generally worse at $0.1 \mathrm{~m}$ when compared to GCP and did not agree with the predicted accuracy. The calibration and accuracy analysis of a low-cost, adaptable mobile mapping system is performed in (Hauser et al., 2016). This system, configured with low-end positioning and mapping sensors, is compared to TLS and high-end MMS data and reports a similar sub-decimetre accuracy in 3D. A more high-end system, Riegl VMX-250, is tested in (Hofmann, Brenner, 2016) by comparing georeferenced GCP along the trajectory of the mobile mapping system. In the initial comparison, the shortest distance between each GCP and the mobile mapping point cloud is computed which had a mean error of $1.5 \mathrm{~cm}$ and standard deviation of $4.1 \mathrm{~cm}$. Additionally, a trajectory adjustment was performed using these GCP which significantly reduced the errors. The accuracy analysis performed in (Puente et al., 2013b) analyses both the relative and absolute accuracy of a high-end Lynx mobile mapper. This paper presents a low-cost calibration standard comprised of multiple spheres on an aluminium block with ac- 
curately calibrated relative distances. The comparison of the reference distances with the measured distances by the mobile mapping system results in a relative accuracy of $10 \mathrm{~mm}$ or less. Furthermore, absolute accuracy levels of $5 \mathrm{~cm}$ are reported on a dataset with optimal GNSS reception quality (PDOP $<2.5$ ). In areas with poorer GNSS reception (PDOP $>4$ ), errors increased up to $30 \mathrm{~cm}$. In our research, a more complete accuracy analysis is performed. On the one hand, both the absolute and relative accuracies are analysed for both a low-end and high-end system. Additionally, these accuracies are predicted and evaluated using three datasets with varying GNSS reception quality (rural, residential and urban area). This results in a more complete and detailed accuracy assessment of both low-end and high-end mobile mapping systems.

\section{METHODOLOGY}

Similarly as performed in (Schaer et al., 2007, Barber et al., 2008, Goel, Lohani, 2015, Craig et al., 2007), we propose a theoretical accuracy model based on the general working principal of a MMS. A point $P_{L}$ is measured by the laser scanner in its own local coordinate system. Using the data from GNSS, IMU and the calibration parameters of the mobile mapping system, $P_{L}$ is transformed to $P_{W}$ in the mapping frame. This global transformation is divided into three consecutive transformations. First, $T_{L}^{I}$ transforms $P_{L}$ from the laser scanner to the IMU coordinate system. This is followed by transformation $T_{I}^{G}$ from the IMU to the GNSS coordinate system. Similarly, $T_{G}^{W}$ performs the transformation to the mapping frame. This global transformation is shown in Figure 3. Each transformation $T$ is defined by a rotation matrix $R$ and translation vector $t$ with the exception of $T_{G}^{W}$ which only contains a translation vector with the RTK position of the GNSS antenna. The global transformation of $P_{L}$ to $P_{W}$ is given by:

$$
\begin{aligned}
P_{W} & =T_{G}^{W} T_{I}^{G} T_{L}^{I} P_{L} \\
& =\left[\begin{array}{cc}
I_{3} & t_{G}^{W} \\
0 & 1
\end{array}\right]\left[\begin{array}{cc}
R_{I}^{G} & t_{I}^{G} \\
0 & 1
\end{array}\right]\left[\begin{array}{cc}
R_{L}^{I} & t_{L}^{I} \\
0 & 1
\end{array}\right] P_{L}
\end{aligned}
$$

In total, this equation is defined by 17 parameters which are summarized in Table 1. While in this equation the GNSS and IMU are shown as two independently operating sensors, their data is combined in a kalman filter in order to compute the SBET of the mobile mapping systems. This results in the rotation angles of the IMU and global positioning of the GNSS antenna respectively in Table 1 . Additionally, each parameter in this table has its own accuracy value which influences the theoretical accuracy level of the mobile mapping system. Using equation (2) for error propagation with uncorrelated variables, the accuracies of $P_{W}$ are calculated in terms of $\sigma_{x}, \sigma_{y}$ and $\sigma_{z}$ (Tellinghuisen, 2001):

$$
\sigma_{f}^{2}=\sum\left(\frac{\partial f}{\partial \beta_{i}}\right)^{2} \sigma_{\beta_{i}}^{2}
$$

with $f$ equal to $P_{W \text { orld }}$ from equation (1), $\beta_{i}$ the list of parameters defined in Table 1 and $\sigma_{\beta_{i}}$ the accuracy of the corresponding parameters.

For this study, the accuracy parameters of the laser scanner and IMU are system dependent. The accuracies of boresight angles, lever-arm offsets and GNSS data are kept constant for both low-end and high-end mobile mapping systems. The values of these parameters are outlined in Table 2, which are based

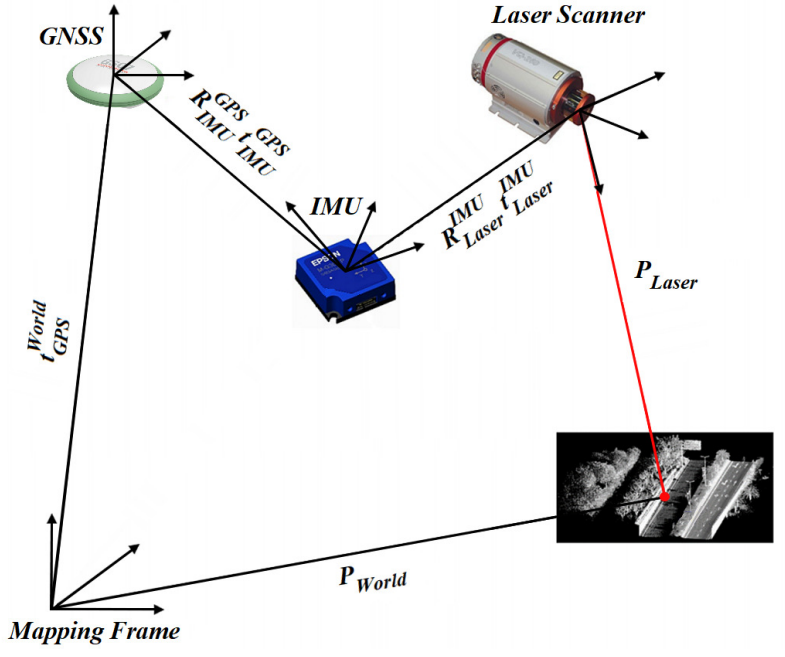

Figure 3. General working principal of mobile mapping systems.

on manufacturer's specifications and previous research (Schaer et al., 2007, Barber et al., 2008, Goel, Lohani, 2015, Craig et al., 2007). Additionally, based on the results from (Ong et al., 2009), a variable GNSS accuracy is included in Table 2 which represents situations with good and poor reception quality. Using these parameters, the theoretical accuracy is predicted for the low-end and high-end system.

\subsection{Low-end MMS: IP-S2 Compact}

The low-end mobile mapping system used in this study is a slightly modified IP-S2 Compact from Topcon, see Figure 1. This system is equipped with 5 KMS511 lidar sensors from SICK of which two sensors are pointed to the left, two to the right and the fifth sensor is facing backwards. The combination and configuration of 5 low-end lidar sensors ensures that the entire environment is captured at a normal driving speed. Each sensor measures at a rate of $38 \mathrm{kHz}$ which brings the total scan rate of the system up to $190 \mathrm{kHz}$. The main disadvantage of these sensors is the limited working range of $1-80 \mathrm{~m}$ and the rapidly degrading accuracy which starts at $8 \mathrm{~mm}$ and increases linearly to about $17 \mathrm{~mm}$ at $20 \mathrm{~m}$. Additionally, this system is equipped with a Ladybug 5 spherical camera instead of the standard Ladybug 3 for higher resolution and better spherical images. In terms of the positioning system only limited information is published about its sensors and calibration by the manufacturer, as is often the case with these low-end systems. This results in a "black box" system in which a limited amount of settings are customizable, restricting the optimisation and upgradability of the system. The remaining system dependent parameters are summarized in Table 3 which are based on manufacturer's specifications and previous research. Using these parameters and the system independent parameters from Table 2, equation (2) computes the absolute accuracy of the system. In order to determine the relative accuracy, the same equation is used with adjusted accuracy values. Because the relative accuracy only depends on the accuracy of the IMU, lidar sensor and the calibration between them, these parameters remain unchanged. All other parameters $\left(t_{I M U}^{G N S}\right.$ and $\left.t_{G N S S}^{W \text { orld }}\right)$ are set to zero. The resulting absolute and relative accuracy are presented in Table 4. 
Table 1. Parameters of global transformation of equation (1).

\begin{tabular}{rll}
\hline Parameter & \multicolumn{1}{c}{ Description } & Source \\
\hline$P_{L}(\theta, d)$ & Coordinates of point $P_{L}$ are defined by scan angle $\theta$ and scanning distance $d$ & Laser scanner \\
$R_{L}^{I}(\alpha, \beta, \gamma)$ & Boresight angles for rotation from laser scanner to IMU coordinate system & System calibration \\
$t_{L}^{I}\left(l_{x}, l_{y}, l_{z}\right)$ & Lever-arm offsets for translation from laser scanner to IMU coordinate system & System calibration \\
$R_{I}^{G}(\omega, \varphi, \kappa)$ & Roll, pith and heading angles for rotation from IMU to GNSS/World coordinate system & IMU \\
$t_{I}^{G}\left(L_{x}, L_{y}, L_{z}\right)$ & Lever-arm offsets for translation from IMU to GNSS coordinate system & System calibration \\
$t_{G}^{W}(x, y, z)$ & Coordinates of GNSS antenna in the global mapping frame & GNSS
\end{tabular}

\subsection{High-end MMS: Lynx mobile mapper SG}

The high-end system used in this study is a modified Lynx system from Optech, see Figure 1. This system is equipped with a dual lidar sensor setup from the Lynx M1 Mobile Mapper. The maximum range of $200 \mathrm{~m}$ and accuracy of $8 \mathrm{~mm}$ ensures accurate lidar data even at large distances. The dual lidar sensor setup captures points at a rate of $500 \mathrm{kHz}$, resulting in a very dense point cloud that contains every detail even at higher driving speeds. Additionally, this system is configured with the camera setup of the Lynx SG Mobile Mapper including four image sensors of $5 \mathrm{MP}$ and a Ladybug 5 spherical camera similar to the low-end system. The positioning system comprises of an Applanix POS LV 420 and a LN-200 IMU which ensures the "surveying-grade" positioning accuracy level. The system dependent parameters are shown in Table 3. Using these parameters, the absolute and relative accuracy of the system are computed in the same manner as the low-end system and are summarized in Table 4.

\subsection{Theoretical accuracy: Low-end vs. High-end}

In good GNSS circumstances, both systems achieve a similar height accuracy of around $0.03 \mathrm{~m}$ while the $2 \mathrm{D}$ accuracy is predicted to be around $0.043 \mathrm{~m}$ and $0.023 \mathrm{~m}$ respectively. This shows that the IMU and lidar accuracy have limited influence on the height accuracy but do have a noticeable influence on the $2 \mathrm{D}$ accuracy. The height accuracy is mainly affected by the height error of the GNSS system as they are of the same order of magnitude. Similarly, the predicted accuracies in poor GNSS circumstances are also dominated by the GNSS accuracy. These results show the importance of a robust and accurate positioning system. In terms of relative accuracy, the low-end and high-end system have a predicted accuracy of $0.023 \mathrm{~m}$ and $0.010 \mathrm{~m}$ respectively caused by the differences in IMU and lidar sensor accuracy.

Table 2. System independent accuracy parameters

\begin{tabular}{cc}
\hline Parameter & Accuracy \\
\hline$\sigma_{\alpha}, \sigma_{\beta}, \sigma_{\gamma}$ & $0.001^{\circ}$ \\
$\sigma_{l_{x}}, \sigma_{l_{y}}, \sigma_{l_{z}}$ & $0.001 \mathrm{~m}$ \\
$\sigma_{L_{x}}, \sigma_{L_{y}}, \sigma_{L_{z}}$ & $0.001 \mathrm{~m}$ \\
$\sigma_{x}, \sigma_{y}$ & $0.014 \mathrm{~m}($ good), $0.07 \mathrm{~m}$ (poor) \\
$\sigma_{z}$ & $0.03 \mathrm{~m}($ good), $0.15 \mathrm{~m}$ (poor) \\
\hline
\end{tabular}

\section{TEST DATASETS}

Six datasets were acquired by both the low-end and high-end MMS in three types of areas: rural, residential and urban. These test areas were carefully selected in order to provide a mix of optimal, good and challenging circumstances for GNSS reception. Each area was recorded by both MMS at the same time to avoid influence of varying GNSS reception and weather conditions. During post-processing, only the GNSS, IMU and DMI data was combined in a kalman filter to compute the SBET. No control points or loop closure techniques were applied in order to quantify the raw absolute accuracy of the mobile mapping data. To perform the accuracy analysis, GCP were materialized in area prior to the data acquisition. These GCP are marked by painted white chequerboard targets measuring 20 by $20 \mathrm{~cm}$ at each intersection and at every $50-75 \mathrm{~m}$ of the trajectory. The coordinates of these GCP were measured with a Leica AX1202 GNSS in RTK mode and a Leica TS15 total station when GNSS reception was not adequate.

\subsection{Rural dataset}

The rural dataset is located $8 \mathrm{~km}$ north of the city centre of Ghent and contains $3.5 \mathrm{~km}$ of roads. This dataset was chosen because of its open areas and limited overhanging vegetation which are considered as optimal circumstances for GNSS reception. The majority of the roads are surrounded by farmlands with all houses at a distance of 10 to $15 \mathrm{~m}$. The other part consists of a regional road with parking spaces and pavement on either side. Residential buildings are located between 5 and $10 \mathrm{~m}$ from the regional road making the space open enough for good GNSS reception. Covering this area are $51 \mathrm{GCP}$, all measured with GNSS.

\subsection{Residential dataset}

Located $3 \mathrm{~km}$ from the city centre of Ghent is the residential dataset containing $6 \mathrm{~km}$ of road. These roads are between 6 and

Table 3. System dependent accuracy parameters for low-end and high-end mobile mapping system

\begin{tabular}{ccc}
\hline Parameter & Low-end & High-end \\
\hline$\sigma_{d}$ & $8 \mathrm{~mm}+1250 \mathrm{ppm}$ & $8 \mathrm{~mm}+10 \mathrm{ppm}$ \\
$\sigma_{\theta}$ & $0.048^{\circ}$ & $0.0045^{\circ}$ \\
$\sigma_{\omega}, \sigma_{\varphi}, \sigma_{\kappa}$ & $0.03^{\circ}, 0.03^{\circ}, 0.09^{\circ}$ & $0.015^{\circ}, 0.015^{\circ}, 0.02^{\circ}$ \\
\hline
\end{tabular}


Table 4. Predicted absolute and relative accuracy of the low-end and high-end system

\begin{tabular}{ccccc}
\hline \multirow{2}{*}{ MMS } & \multicolumn{3}{c}{ Absolute accuracy } & Relative accuracy \\
& Height error* & 2D error* & 3D error* & 3D error \\
\hline Low-end & $0.034 \mathrm{~m} / 0.151 \mathrm{~m}$ & $0.043 \mathrm{~m} / 0.107 \mathrm{~m}$ & $0.054 \mathrm{~m} / 0.185 \mathrm{~m}$ & $0.023 \mathrm{~m}$ \\
High-end & $0.031 \mathrm{~m} / 0.150 \mathrm{~m}$ & $0.023 \mathrm{~m} / 0.101 \mathrm{~m}$ & $0.038 \mathrm{~m} / 0.181 \mathrm{~m}$ & $0.010 \mathrm{~m}$ \\
\hline
\end{tabular}

${ }^{*}$ (good GNSS reception quality) / (poor GNSS reception quality)

$8 \mathrm{~m}$ wide with parking spaces and pavement on one or either side. While in most streets the residential buildings are located at a distance of $5 \mathrm{~m}$ from the pavement, there are a few streets with a more urban character as the buildings are built right along the pavement. Although the residential buildings are closer to the road in comparison with the rural dataset, it is expected that the dense overhanging vegetation in this dataset will have more influence on the GNSS reception quality. This is especially the case in this dataset where a part is heavily occluded by tall trees of $20 \mathrm{~m}$ and higher which will limit the accuracy of the MMS. Covering this dataset are 67 GCP all measured with GNSS. As these GCP were measured 2 months before the mobile mapping acquisition, the trees had no leaves yet making accurate GNSS measurements possible in these areas.

\subsection{Urban dataset}

The urban dataset is located right in the city centre and contains $3 \mathrm{~km}$ of roads. While the majority are two-way streets with pavement on either side, there are a lot of smaller one-way streets with residential buildings close to each other and adjacent to the road. In combination with some high vegetation, this creates difficult and challenging circumstances for GNSS to operate properly and accurate. Covering the dataset are 80 GCP of which half are measured accurately with GNSS while the remaining were measured with a total station when GNSS reception was limited.

\section{EXPERIMENTS}

The theoretical absolute and relative accuracy from Table 4 are validated against ground truth measurements for the low-end and the high-end mobile mapping system. Both systems are analysed in the three different datasets with varying GNSS reception quality.

\subsection{Absolute accuracy}

In order to quantify the absolute accuracy of the mobile mapping systems, the raw mobile mapping data is compared to the GCP in each area in terms of vertical, horizontal and 3D accuracy. The coordinates of the GCP are extracted from the mobile mapping data using 3DM Content Manager from Orbit (Orbit GeoSpatial Technologies, 2019). In this software package, the operator marks the GCP in the image, after which the coordinates are computed by projecting the image point onto the point cloud. On the one hand, the combination of using images and point cloud data has the advantage that the GCP only needs to be visible in one image in order to determine its coordinates. On the other hand, good alignment between the images and point cloud data is necessary. A misalignment of either results in additional systematic errors which compromises the accuracy of the results. This issue does not arise when marking the GCP directly on the point cloud data using the intensity values. However, this method requires a high point density to accurately mark the GCP, which is not possible on the low-end datasets as is shown by Figure 4. Table 5 summarizes the height, 2D and 3D errors of the comparison for each dataset and mobile mapping system. Errors due to long GNSS outage were excluded from these results as GNSS outage is not accounted for in the theoretical model and these outliers have a significant influence on the statistics in Table 5.

In terms of height error, both the low-end and high-end system agree with the predicted accuracy in the rural area. In contrast, the height errors double and triple for the high-end and low-end system respectively in the residential and urban dataset. As a result, the achieved accuracies do not longer correspond to the predicted values for good GNSS circumstances. However, they do fall within the predicted interval for poor GNSS reception. With a RMSE of $0.095 \mathrm{~m}$ and 0.103 for the low-end system in the residential and urban dataset respectively, these errors are twice the accuracy of the high-end system in these datasets with a RMSE of $0.053 \mathrm{~m}$ and 0.041 respectively.

In terms of 2D error, the theoretical model fails to correctly predict the accuracy for either system in good GNSS circumstances. With a RMSE of $0.107 \mathrm{~m}, 0.130 \mathrm{~m}$ and 0.240 for the three low-end datasets, only the rural dataset barely meets the interval for poor GNSS reception quality. In contrast, the highend systems results in a RMSE of $0.039 \mathrm{~m}, 0.064 \mathrm{~m}$ and 0.074 $\mathrm{m}$ for the rural, residential and urban dataset respectively. Compared to the low-end system, these results are much lower and do agree comfortably with the predicted accuracy in poor GNSS conditions. Similar as for the height errors, the $2 \mathrm{D}$ errors of the low-end system are around twice or three times as large. The same trend and conclusions can be drawn from the results of the $3 \mathrm{D}$ errors as they do not agree with the theoretical model.

In general the high-end system outperforms the low-end system in height, 2D and 3D accuracy. This is also shown in Figures 5, 6 and 7 which visualize the $3 \mathrm{D}$ errors in the rural, residential and urban dataset respectively. As these results show, both mobile mapping systems perform best in the rural area which is mainly due to the wide open space resulting in better GNSS reception. However this is not always the case for the low-end system which shows a decrease in accuracy in the lower left part of the dataset with an error up to $30 \mathrm{~cm}$. It is unclear what caused this as this rural road is surrounded by wide open farm lands and no houses or trees are present within 100 meters. When compared to the high-end dataset, this accuracy decrease is not observed. The largest errors in this dataset of around $10 \mathrm{~cm}$ are due to obstacles such as houses and trees in those areas. In the residential dataset, the errors increase for both systems. While they perform reasonably well in the majority of the dataset, both systems fail to sustain their accuracy in the lower right part of the dataset. This error increase is mainly caused by the limited or loss of GNSS reception due to large overhanging trees in this area. As both systems experience GNSS outage, it is clear from these results that the 
Table 5. Summary of height, 2D and 3D errors for the low-end and high-end mobile mapping system in the different datasets.

\begin{tabular}{|c|c|c|c|c|c|c|c|c|c|c|}
\hline \multirow{2}{*}{ MMS } & \multirow{2}{*}{ Dataset } & \multicolumn{3}{|c|}{ Height error } & \multicolumn{3}{|c|}{$2 D$ error } & \multicolumn{3}{|c|}{ 3D error } \\
\hline & & Mean & Min/Max error & RMSE* & Mean & Max error & RMSE* & Mean & Max error & RMSE* \\
\hline \multirow{3}{*}{ Low-end } & Rural & $-0.024 \mathrm{~m}$ & $0.137 \mathrm{~m}$ & $0.032 \mathrm{~m}$ & $0.103 \mathrm{~m}$ & $0.268 \mathrm{~m}$ & $0.107 \mathrm{~m}$ & $0.109 \mathrm{~m}$ & $0.272 \mathrm{~m}$ & $0.112 \mathrm{~m}$ \\
\hline & Residential & $-0.030 \mathrm{~m}$ & $2.319 \mathrm{~m}$ & $0.095 \mathrm{~m}$ & $0.121 \mathrm{~m}$ & $2.211 \mathrm{~m}$ & $0.130 \mathrm{~m}$ & $0.145 \mathrm{~m}$ & $2.933 \mathrm{~m}$ & $0.161 \mathrm{~m}$ \\
\hline & Urban & $0.010 \mathrm{~m}$ & $-0.500 \mathrm{~m}$ & $0.103 \mathrm{~m}$ & $0.200 \mathrm{~m}$ & $0.789 \mathrm{~m}$ & $0.240 \mathrm{~m}$ & $0.219 \mathrm{~m}$ & $0.934 \mathrm{~m}$ & $0.261 \mathrm{~m}$ \\
\hline \multirow{3}{*}{ High-end } & Rural & $0.004 \mathrm{~m}$ & $0.047 \mathrm{~m}$ & $0.024 \mathrm{~m}$ & $0.037 \mathrm{~m}$ & $0.118 \mathrm{~m}$ & $0.039 \mathrm{~m}$ & $0.044 \mathrm{~m}$ & $0.125 \mathrm{~m}$ & $0.046 \mathrm{~m}$ \\
\hline & Residential & $-0.040 \mathrm{~m}$ & $-0.231 \mathrm{~m}$ & $0.053 \mathrm{~m}$ & $0.058 \mathrm{~m}$ & $0.266 \mathrm{~m}$ & $0.064 \mathrm{~m}$ & $0.076 \mathrm{~m}$ & $0.305 \mathrm{~m}$ & $0.084 \mathrm{~m}$ \\
\hline & Urban & $-0.024 \mathrm{~m}$ & $-0.109 \mathrm{~m}$ & $0.041 \mathrm{~m}$ & $0.068 \mathrm{~m}$ & $0.193 \mathrm{~m}$ & $0.074 \mathrm{~m}$ & $0.078 \mathrm{~m}$ & $0.222 \mathrm{~m}$ & $0.085 \mathrm{~m}$ \\
\hline
\end{tabular}

$*$ RMSE $=$ Root Mean Square Error

high-end system performs more robustly and limits the maximum error to $40 \mathrm{~cm}$. In contrast, the low-end mobile mapping system drifted a lot more which resulted in 3D errors up to 2 - 3 meters. These results show that the high-end system is much more consistent in challenging circumstances especially in areas with loss of GNSS reception. This is mainly due to the high-end IMU and DMI which accurately determine the position of the MMS and are less prone to drift in these challenging circumstances. Similar results are observed in the urban dataset as the low-end system struggled to sustain its accuracy in the narrow urban streets. The loss of accuracy in these areas is mainly caused by the urban canyon effect. This influences the accuracy of the low-end system more than the high-end system, as is shown by the maximum error of $0.934 \mathrm{~m}$ and 0.222 $\mathrm{m}$ respectively. One possible reason why the high-end system outperforms the low-end system in these circumstances is the position of the GNSS sensor on the mobile mapping system. When comparing the both systems in Figure 1, it can be seen that the GNSS sensor is placed around $1 \mathrm{~m}$ higher on the highend system. This results in less influence of the urban canyon effect and a more robust performance in these challenging circumstances.

As these results show, the theoretical accuracy model of the mobile mapping system does not fit the observations made in the different datasets which could be due to a few reasons. On the one hand, because no calibration parameters are published by the manufacturer, these parameters were selected based on previous research. It is possible that these accuracies are an overestimation of the true accuracy values of the system. Additionally, the same GNSS accuracy is used for both systems while the results show that, because of varying mounting positions, they will perform differently. On the other hand, using the combination of images and point cloud in order to extract the coordinates of the GCP introduces additional errors that are not included in the theoretical model. Repeated marking of a GCP in a single images, for example, results in a marking error of around $8 \mathrm{~mm}$ in 2D for either system as they use the same camera. Additionally, marking the same GCP in consecutive camera positions resulted in a variation in 2D coordinates of $5 \mathrm{~cm}$ and $2 \mathrm{~cm}$ for the low-end and high-end system respectively. Furthermore, the low-end system also showed a slight misalignment between lidar and image data when the point cloud is projected onto the image. These results show that a recalibration, especially of the low-end system, is necessaries in order to perform a proper accuracy analysis using this marking method. Further research should focus on correctly documenting the accuracy parameters used for the theoretical accuracy model and a robust analysis method that eliminates the additional errors due to the marking method of the GCP. Additionally, an improved variable GNSS parameter will be investigated which takes the mounting position and occluding objects in the vicinity into account in order to make a more accurate prediction.
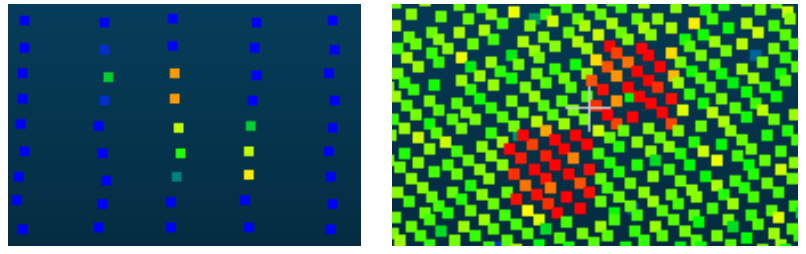

Figure 4. GCP in the point cloud data coloured based on the intensity value of the low-end system (left) and high-end system (right)

Table 6. Summary of the relative accuracy experiment.

\begin{tabular}{cccc}
\hline MMS & Mean & Std. dev. & RMSE \\
\hline Low-end & $-0.3 \mathrm{~mm}$ & $15.7 \mathrm{~mm}$ & $15.6 \mathrm{~mm}$ \\
High-end & $8.0 \mathrm{~mm}$ & $4.6 \mathrm{~mm}$ & $9.2 \mathrm{~mm}$ \\
\hline
\end{tabular}

\subsection{Relative accuracy}

The predicted relative accuracy is validated by comparing 100 control distances in the mobile mapping point clouds. These control distances are measured in a ground truth point cloud captured with a Leica P30 terrestrial laser scanner and consists of an urban street with adjacent houses on both side. First, 100 control points are manually marked on the facades of the houses on one side of the street. Next, opposite of each control point, a second control point is measured on the facade of the house on the other side of the street forming a pair. This results in 100 pairs which are used to compute the 100 control distances. The same pair of points are measured in the mobile mapping datasets in order to compute the distances. Because the point pairs are located directly opposite of each other, this eliminates the influence of the GNSS accuracy to validate the theoretical relative accuracy of the mobile mapping system.

The results of this experiment are shown in Table 6. The lowend system produced a mean error of $-0.3 \mathrm{~mm}$, standard deviation of $15.7 \mathrm{~mm}$ and RMSE of $15.6 \mathrm{~mm}$ which agrees very well with the predicted accuracy of $18 \mathrm{~mm}$. In contrast, the high-end system has a mean error of $8 \mathrm{~mm}$, standard deviation of $4.5 \mathrm{~mm}$ and a RMSE of $9.2 \mathrm{~mm}$. Although this agrees with the predicted accuracy of $9.5 \mathrm{~mm}$, the mean error of $8 \mathrm{~mm}$, which is expected to be close to $0 \mathrm{~mm}$, indicates a systematic error in the system. Without further research, the cause of this error is unknown but can possibly be corrected by recalibrating the system.

\section{CONCLUSION}

This paper presents a theoretical accuracy model to predict the absolute and relative accuracy of mobile mapping systems. The 

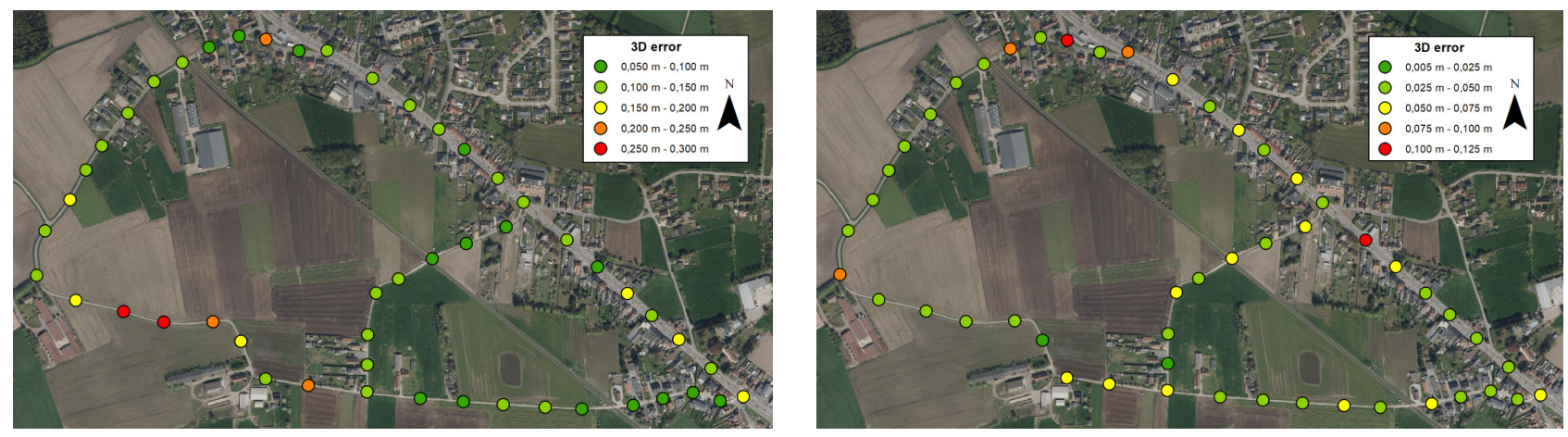

Figure 5. 3D errors of GCP in rural dataset for the low-end system (left) and high-end system (right).
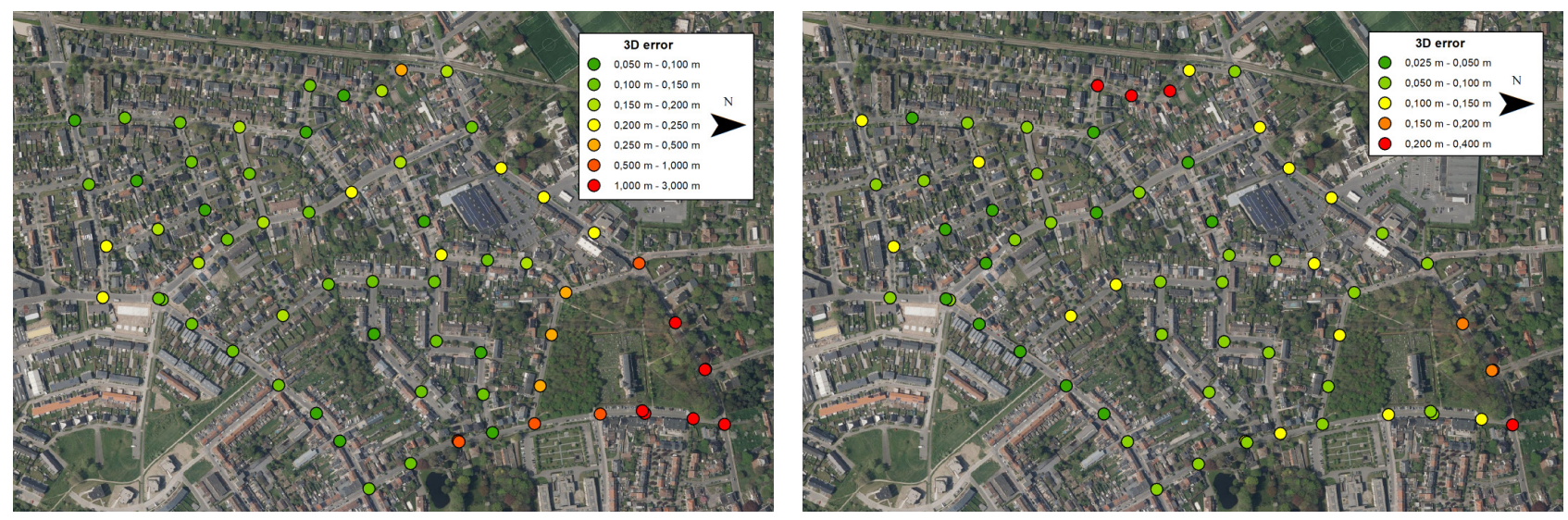

Figure 6. 3D errors of GCP in residential dataset for the low-end system (left) and high-end system (right).
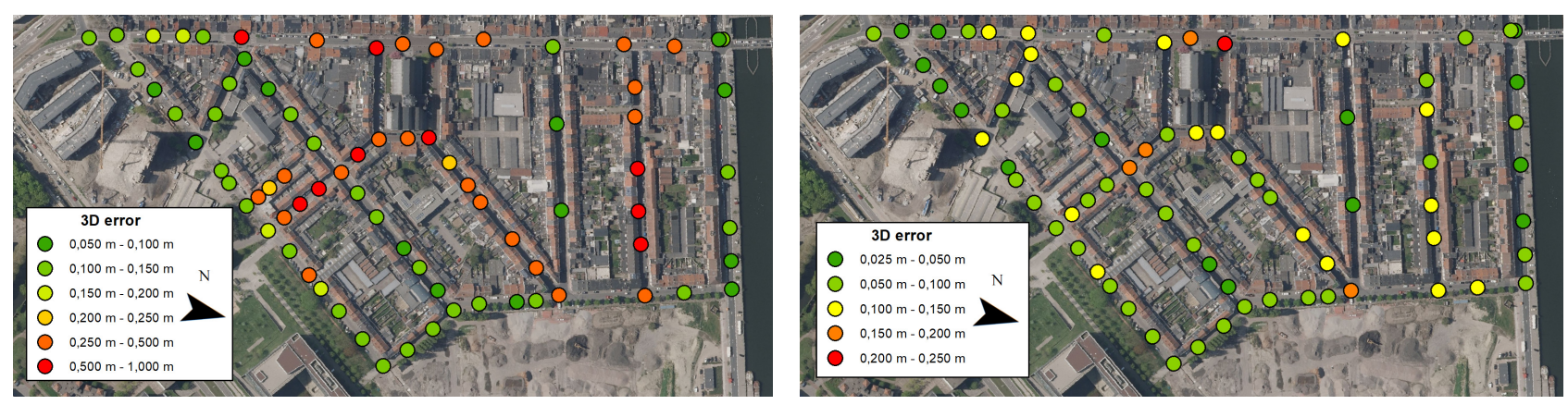

Figure 7. 3D errors of GCP in urban dataset for the low-end system (left) and high-end system (right).

theoretical model includes a varying accuracy for the GNSS and is used to predict the accuracies of a low-end and high-end mobile mapping system. Three test datasets are captured with both a low-end and high-end mobile mapping system which included different environments with varying GNSS reception quality. The absolute accuracy is validated using GCP, measured with GNSS and total station. Additionally, the relative accuracy is verified using control distances from a ground truth point cloud, captured with a terrestrial laser scanner.

The results of our experiments show that the theoretical model fails to provide a correct prediction of the absolute accuracy. While good GNSS reception is expected in the entire rural dataset, only the height error agreed with the predicted values for both systems while the 2D RMSE was up to twice as large as expected. In the other datasets the errors increased as occlud- ing objects deteriorated the GNSS accuracy. While the highend system was within the predicted intervals for poor GNSS reception, the results of the low-end system were not. On the one hand, this is possibly due to overestimation of the accuracy parameter used in the theoretical model. On the other hand, the marking method which uses the combination of image and point cloud data introduces additional errors that influence the accuracy analysis results considerably. When comparing the lowend and the high-end system, the latter always outperformed the former. The high-end system especially performed more consistent, accurate and robust in challenging circumstances such as urban canyons and densely forested areas. This performance difference is partly due to more accurate sensors and calibration but also because of the higher mounting position of the GNSS antenna on the high-end mobile mapping system. This higher mounting position reduces the influence of the urban canyon 
effect which results in a more robust performance in these challenging circumstances. Further research will focus on correctly documenting the theoretical parameters used for the theoretical accuracy model, a robust analysis method that eliminates the additional errors from the marking method and an improved variable GNSS accuracy parameter which takes into account the mounting position and occluding objects in the vicinity.

\section{ACKNOWLEDGEMENTS}

This project received funding from the FWO research foundation (under FWO PhD SB fellowship 1S87120N) and the research group Geomatics of the Department of Civil Engineering, TC Construction at the KU Leuven in Belgium. We would also like to thank surveying company Teccon for the acquisition of the mobile mapping datasets.

\section{REFERENCES}

Ajmar, A., Balbo, S., Boccardo, P., Tonolo, F. G., Piras, M., Princic, J., 2013. A Low-Cost Mobile Mapping System ( LCMMS ) for field data acquisition : a potential use to validate aerial/satellite building damage assessment. International Journal of Digital Earth, 6(2), 103-123.

Barber, D., Mills, J., Smith-Voysey, S., 2008. Geometric validation of a ground-based mobile laser scanning system. ISPRS Journal of Photogrammetry and Remote Sensing, 63(1), 128-141.

Bitenc, M., Lindenbergh, R., Khoshelham, K., van Waarden, A. P., 2011. Evaluation of a LIDAR land-based mobile mapping system for monitoring sandy coasts. $R e$ mote Sensing, 3, 1472-1491.

Craig, G., Glennie, C., Ph, D., Eng, P., 2007. Rigorous 3D error analysis of kinematic scanning LIDAR systems. Journal of Applied Geodesy, 1, 147-157.

Goel, S., Lohani, B., 2015. Relative Contribution and Effect of Various Error Sources on the Performance of Mobile Mapping System (MMS). Journal of the Indian Society of Remote Sensing, 43(3), 639-645.

Hauser, D., Glennie, C., Brooks, B., 2016. Calibration and Accuracy Analysis of a Low-Cost Mapping-Grade Mobile Laser Scanning System. Journal of Surveying Engineering, 142(4).

Hofmann, S., Brenner, C., 2016. Accuracy assessment of mobile mapping point clouds using the existing environment as terrestrial reference. The International Archives of the Photogrammetry, Remote Sensing and Spatial Information Sciences, XLI-B1, 601-608.

Hwang, J., Yun, H., Jeong, T., Suh, Y., Huang, H., 2013. Frequent Unscheduled Updates of the National Base Map Using the Land-Based Mobile Mapping System. Remote sensing, 5, 2513-2533.

Kaartinen, H., Hyyppä, J., Kukko, A., Jaakkola, A., Hyyppä, H., 2012. Benchmarking the performance of mobile laser scanning systems using a permanent test field. Sensors, 12, 12814-12835.
Kersting, A. P., Friess, P., 2016. Post-mission quality assurance procedure for survey-grade mobile mapping systems. International Archives of the Photogrammetry, Remote Sensing and Spatial Information Sciences - ISPRS Archives, 2016-Janua(July), 647-652.

Ong, R. B., Petovello, M. G., Lachapelle, G., 2009. Assessment of GPS/GLONASS RTK Under Various Operational Conditions. Proceedings of the 22nd International Technical Meeting of The Satellite Division of the Institute of Navigation, 3297-3308.

Orbit GeoSpatial Technologies, 2019. 3DM Content Manager.

Puente, I., Arias, P., González-Jorge, H., MartínezSánchez, J., Arias, P., 2013a. Review of mobile mapping and surveying technologies. Measurement, 46(7), 21272145 .

Puente, I., González-Jorge, H., Riveiro, B., Arias, P., 2013b. Accuracy verification of the Lynx Mobile Mapper system. Optics and Laser Technology, 45, 578-586.

Sairam, N., Nagarajan, S., Ornitz, S., 2016. Development of Mobile Mapping System for 3D Road Asset Inventory. Sensors, 16(3).

Schaer, P., Skaloud, J., Landtwing, S., Legat, K., 2007. Accuracy estimation for laser point cloud including scanning geometry. International Archives of the Photogrammetry, Remote Sensing and Spatial Information Sciences - ISPRS Archives, 36(5), N/A.

Tellinghuisen, J., 2001. Statistical error propagation. The Journal of Physical Chemistry A, 105(15), 3917-3921.

Toschi, I., Rodríguez-Gonzálvez, P., Remondino, F., Minto, S., Orlandini, S., Fuller, A., 2015. Accuracy evaluation of mobile mapping systems with advanced statistical methods. The International Archives of the Photogrammetry, Remote Sensing and Spatial Information Sciences, XL(5), 245-253. 